\title{
Caracterización de los deportistas Paralímpicos de Goalball Chilenos y asociación entre variables Antropométricas, de Composición Corporal y Fuerza en el Lanzamiento del Balón Characterization of Chilean Goalball Paralympic athletes and association between Anthropometric variables, Body Composition and Strength in Ball Throwing \\ *Luis Felipe Castelli Correia de Campos, **Kevin Campos-Campos, **Juan Gajardo-Oñate \\ **Cristian Eduardo Luarte Rocha, ${ }^{* * *}$ Luiz Gustavo Teixeira Fabricio dos Santos, ${ }^{* * * *}$ Raúl Smith Plaza, ${ }^{* * * * *}$ Jerusa Petróvna Resende Lara \\ *Universidad del Bío-Bío (Chile), **Universidad San Sebastián (Chile), ***Comitê Paralímpico Brasileiro (Brasil), ****Universidad
} del Desarrollo (Chile), *****Universidade Federal do Paraná (Brasil)

Resumen. Objetivo: Identificar la asociación y relación entre las variables de fuerza máxima, potencia muscular, medidas antropométricas y composición corporal con el lanzamiento del balón de Goalball. Método: Nueve jugadores varones de Goalball, pertenecientes a dos equipos amateur de la región del Biobío. Se registraron medidas antropométricas de Longitud de Miembros Superiores (CMS) y Envergadura (ENV), Porcentaje de Grasa Corporal (\%GC), test físicos correspondientes a una repetición máxima (1RMe) en press banca, Test de lanzamiento de balón medicinal (AMB) para relacionar las variables con el test de lanzamiento (TLAN) en Goalball. Resultados: Los principales resultados observados fueron correlaciones muy altas, inversamente proporcionales y estadísticamente significativas entre TLAN y $1 \mathrm{RMe}(\mathrm{r}=-.80, \mathrm{p}=.008)$, entre ENV y $1 \mathrm{RMe}(\mathrm{r}=-.74, \mathrm{p}=.021), \mathrm{CMS}$ y $1 \mathrm{RMe}(\mathrm{r}=-.72, \mathrm{p}=.027)$ y correlaciones muy altas y significativas entre AMB y $1 \mathrm{RMe}(\mathrm{r}=.89, \mathrm{p}=.001)$. Conclusión: En relación a los datos obtenidos en el presente estudio, existe una correlación muy alta y significativa entre fuerza máxima estimada y menores tiempos en el lanzamiento del Goalball, lo que refleja que la fuerza es una cualidad determinante en la acción ofensiva, no así las medidas antropométricas, ya que no influyeron en la eficiencia del lanzamiento.

Palabras Clave: Goalball, Entrenamiento Deportivo, Antropometría, Deporte Paralímpico, Fuerza.

\begin{abstract}
Objective: To identify the association and relationship between the variables of maximum strength, muscle power, anthropometric measurements, and body composition with Goalball ball throwing. Method: Nine male Goalball players belonging to two amateur teams from the Biobío region. Anthropometric measures of Length of Upper Members (CMS) and Wingspan (ENV), Body Fat Percentage (\% $\mathrm{GC}$ ), and physical tests corresponding to one maximum repetition (1RMe) in bench press and medical ball throw Test (AMB) were recorded in order to relate the variables with the throwing test (TLAN) in Goalball. Results: Very high inversely proportional and statistically significant correlations between TLAN and $1 \mathrm{RMe}(\mathrm{r}=-.80, \mathrm{p}=.008)$, between VNS and 1RMe $(\mathrm{r}=-.74$, $\mathrm{p}=.021)$, and between CMS and 1RMe ( $\mathrm{r}=-.72, \mathrm{p}=.027)$ were observed, as well as very high and significant correlations between AMB and 1RMe $(\mathrm{r}=.89, \mathrm{p}=.001)$. Conclusion: In relation to the data obtained in the present study, there is a very high and significant correlation between estimated maximum strength and shorter times in the launch of the Goalball, which shows that strength is a determining quality in offensive actions, whereas anthropometric measures are not, since they did not influence throwing efficiency.
\end{abstract}

Keywords: Goalball, Sports Training, Anthropometry, Sport Paralympic, Strength.

\section{Introducción}

El Goalball es uno de los principales deportes colectivos paralímpico practicado por atletas con ceguera y baja visión, siendo integrado en los Juegos Paralímpicos desde 1976. Para el desarrollo del juego participan dos equipos de tres atletas cada uno, con el objetivo principal que, el balón, el cual produce estímulos sonoros, cruce rodando la línea de gol del equipo contrario mientras el otro equipo intenta impedirlo (Sanz, \& Reina, 2014).

Así, en relación a la demanda del esfuerzo presentado por los deportistas, la potencia muscular principalmente para las acciones ofensivas como el lanzamiento del balón, la velocidad de reacción para acciones defensivas y la resistencia de la fuerza, son destacadas debido a que las acciones son realizadas enumeradas veces (Da Rocha, 2007; Pereira, Simöes, \& Gaviäo, 2012; Gómez \& Tosim, 2016).

Según Corredeira, Lima \& Botelho(2009), la fuerza es una cualidad determinante en el desarrollo del juego, especialmente en los lanzamientos, donde un lanzamiento rápido aumenta significativamente las posibilidades de marcar, por

Fecha recepción: 19-06-19. Fecha de aceptación: 08-11-19 Luis Felipe Castelli Correia de Campos tcastelli@ubiobio.cl lo tanto, un entrenamiento de la fuerza, principalmente en su manifestación como potencia, es importante para la mejora de la velocidad en los lanzamientos (Hernández, Lara \& Iturriaga, 2017; Morato, 2012; Gómez \& Tosim, 2016).

Se han desarrollado varios estudios para comprender los mecanismos y variables que pueden interferir en el lanzamiento en diferentes modalidades deportivas (Barrera et al., 2020; Jimenez-Olmedo, Espina-agullo \& Manchado, 2017; Guzmán, Fonseca \& Jiménez, 2016; Frutos, 2014). Autores como Bowerman, Davis, Ford \& Nichols (2011), estudiaron las fases del lanzamiento de Goalball en función de la velocidad, encontrando una mayor velocidad empleada en el lanzamiento en giro, enfatizando que este dato está registrado sin tener en cuenta las características de los jugadores que lanzan el balón.

Respecto al perfil Cineantropométrico de la modalidad, Rodrigues y Bastos (2007) determinaron que los deportistas practicantes de Goalball en la liga brasileña presentaban sobrepeso y malos hábitos nutricionales, lo que determinaba un perfil nutricional y antropométrico poco adecuado para la práctica del deporte, influenciando negativamente en el rendimiento deportivo.

En tanto, las variables que pueden presentar una mejor relación con el lanzamiento del balón, factor determinante para el éxito durante los partidos, aún son desconocidas. En 
ese sentido, el objetivo de esta investigación es identificar el perfil de los atletas de Goalball chilenos como también identificar la asociación y relación entre las variables antropométricas, de composición corporal y fuerza con el lanzamiento del balón de Goalball.

\section{Material y Métodos}

\section{Muestra}

Participaron del presente estudio nueve atletas varones $(36,3 \pm 13,3$ años, $80,3 \pm 5,7 \mathrm{Kg}, 170,8 \pm 3,5 \mathrm{~cm})$ y clasificación oftalmológica B1 (n=3), B2 (n=3) y B3 ( $=3)$. Todos pertenecientes a dos equipos de Goalball de la región del Biobío/ Chile y que han participado en competiciones a nivel nacional un mínimo de 3 años. Siguiendo las normas establecidas para investigaciones con seres humanos, todos los atletas firmaron el consentimiento libre e informado. El protocolo de investigación fue revisado y aprobado por el Comité Ético Científico de la Universidad Autónoma de Chile y desarrollado siguiendo lo expuesto en la Declaración de Helsinki.

\section{Procedimiento}

Para el desarrollo del estudio los deportistas fueron sometidos a las mediciones antropométricas de peso corporal (balanza Bioland $^{\circledR}$, modelo EF912), con precisión de $0,1 \mathrm{~kg}$, de la talla (Estadiómetro portátil Sanny ${ }^{\circledR}$, modelo ES-2060) con precisión en $0,1 \mathrm{~cm}$ y de los pliegues cutáneos; tricipital, bicipital, subescapular y supra ilíaco con el adipómetro (Harpender $^{\circledR}$, modelo C-136) con precisión en 0,2 mm. Todos los procedimientos fueron recolectados de acuerdo al protocolo propuesto por ISAK(2011).

Para las medidas antropométricas de envergadura (ENV) y longitud extremidad superior (CMS) se utilizó la cinta métrica (Lufkin ${ }^{\circledR}$, modelo W606PM) y protocolo de acuerdo a lo propuesto por Guedes \& Guedes(2006). Para calcular la densidad corporal se utilizó la fórmula propuesta por Durnin and Womersley (1974) y el porcentaje de Grasa Corporal (\%GC) la ecuación de Siri(1956).

En relación a los test motores, se utilizó el test de Press de Banca para estimar la fuerza máxima de los deportistas en una repetición máxima (1RMe). Todos los procedimientos para la realización del test y la ecuación para el cálculo de 1RMe fueron realizados de acuerdo a lo propuesto por Amarante do Nascimento, Serpeloni-Cyrino, Yuzo-Nakamura, Romanzini, Cardoso-Pianca, \& Queiróga (2007).

Se realizó el test de lanzamiento del balón medicinal (ABM) con los atletas sentados en suelo, según protocolo propuesto por Matínez (2002). Para el desarrollo del test se utilizó el balón medicinal de $2 \mathrm{~kg}$ y los atletas realizaron tres intentos siendo registrado el lanzamiento con la mayor distancia lograda en metros.

Finalmente, los deportistas fueron convocados para realizar el lanzamiento frontal del balón de Goalball, en donde se calculó el tiempo de la trayectoria del balón hasta que logró pasar la línea del gol adversaria (TLAN). Como procedimiento padrón fue instalada una cinta adhesiva en el suelo con relieve para identificación del punto inicial. El atleta fue orientado a mantener una de las rodillas en suelo en la distancia del máximo de flexión de hombro hasta que pierda el contacto con el balón, considerando marca cero e inicio de tiempo.
Para auxiliar en la orientación del lanzamiento, se ubicó un evaluador emitiendo el sonido (aplauso) del otro lado de la cancha (en la línea del gol adversario). Para que el lanzamiento fuese registrado, el balón no podría exceder 1 metro hacia los lados del evaluador. El tiempo demarcado en segundos, se registra desde el momento en que el atleta perdió el contacto con el balón en la línea de demarcación inicial, hasta el momento en que el balón cruza la línea de gol adversaria. Fue utilizado el recurso de filmación (Cámara Digital Deportiva Gopro Hero 7 Black) bajo la observación de un evaluador experto.

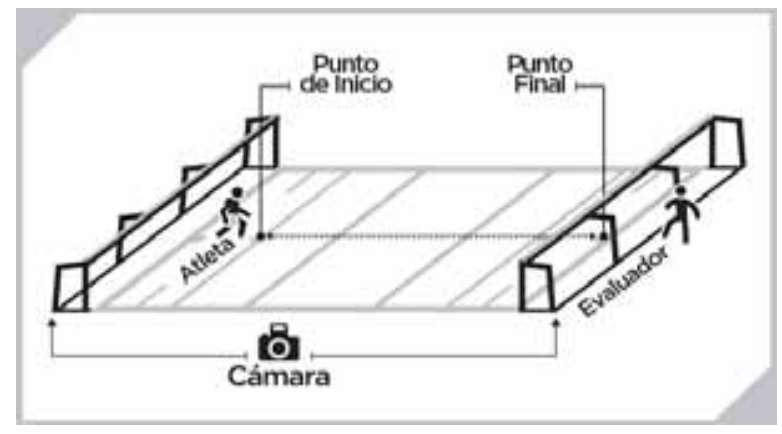

Figura 1. Test Lanzamiento balón de Goalball (TLAN)

\section{Análisis estadístico}

Las variables del presente estudio fueron presentadas en promedio, desviación estándar (de), mínimo (min), máximo (máx) e intervalo de confianza (IC95\%). Para comprobar la normalidad de los datos se utilizó el test Shapiro-Wilk. Confirmando que los datos presentaban distribución normal, se utilizó el procedimiento de correlación de Pearson entre las variables ENV, CMS, \%GC, 1RMe, AMB y TLAN. Los valores de correlación fueron determinados a partir del propuesto por Hopkins(2000).

Para la relación de causa y efecto fue utilizado el procedimiento de regresión lineal simple, en lo cual las variables independientes (ENV, CMS, \%GC, 1RMe, AMB) fueron correlacionadas con la variable dependiente (TLAN). El análisis estadístico y su representación gráfica se realizó mediante el software GraphPad Prism ${ }^{\circledR}$ 7.0. Todos los test adoptaron un nivel de significancia de pe» 0,05 .

\section{Resultados}

Los valores de promedio, desviación estándar, mínimo, máximo y los intervalos de confianza de las variables antropométricas, de composición corporal y del rendimiento motor de los atletas son presentadas en la tabla 1 .

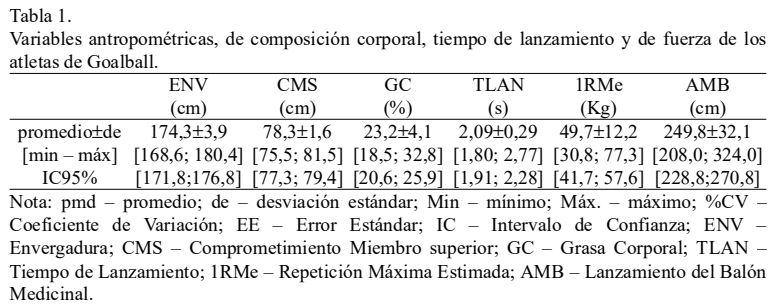
Medicinal.

Las correlaciones entre las variables son presentadas en la figura 2. Fueron observadas correlaciones muy altas, inversamente proporcionales y significativas entre TLAN con $1 \mathrm{RMe}(\mathrm{r}=-.80, \mathrm{p}=.008), \operatorname{ENV}$ y $1 \mathrm{RMe}(\mathrm{r}=-.74, \mathrm{p}=.021)$, CMS y $1 \mathrm{RMe}(\mathrm{r}=-.72, \mathrm{p}=0.027)$ y correlaciones muy altas $\mathrm{y}$ 
significativas entre AMB y $1 \mathrm{RMe}(\mathrm{r}=.89, \mathrm{p}=.001)$.

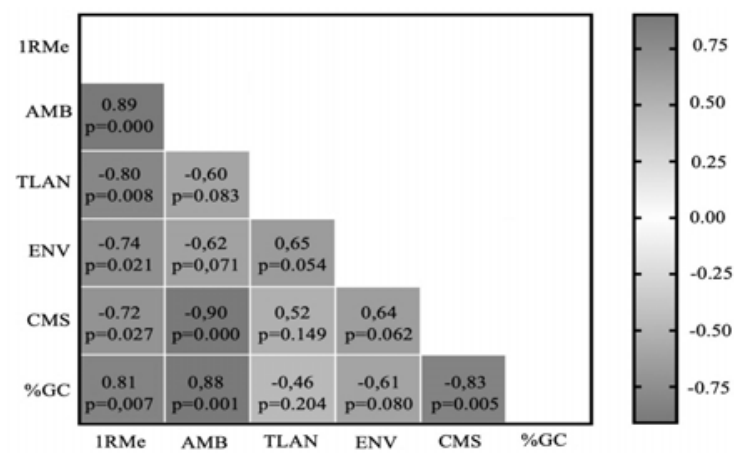

Figura 2. Correlación de Pearson entre las variables antropométricas (ENV, CMS), de composición corporal, de fuerza y el tiempo de lanzamiento (TLan).

Nota: 1RMe - Repetición Méxim Estimada; AMB - Lanzamiento del Balón Medicinal; TLAN - Timp - de Lanzamiento; ENV - Envergadura; CMS - Comprotimiento Mienbro superior; GC - Grasa Corporal

Para la relación de causa y efecto entre las variables independientes (ENV, CMS, \%GC, 1RMe, AMB) con la variable dependiente (TLAN) fue utilizado el procedimiento de regresión lineal simple de acuerdo a lo presentado en la figura 3. Se observó valores más elevados de determinación entre TLAN y $1 \mathrm{Rme}\left(\mathrm{R}^{2}=.64\right)$ y TLAN y ENV $\left(\mathrm{R}^{2}=.43\right)$.
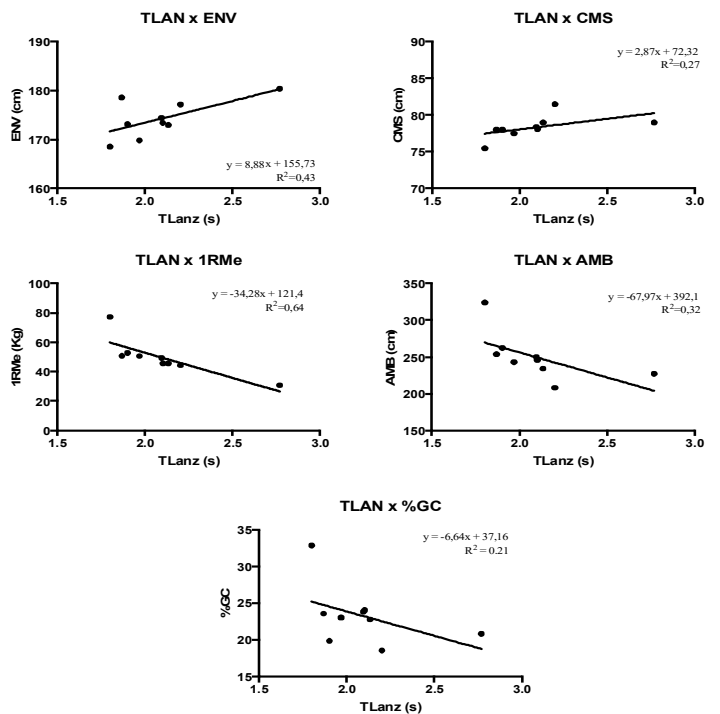

Figura 3. Análisis de regresión lineal simple de las variables antropométricas, de composición Figura 3. Análisis de regresión lineal simple de las variables antropométricas, de composición
corporal y de los test de rendimiento motor con el Tiempo de lanzamiento de Goalball corporal y de los test de rendimiento motor con el Tiempo de lanzamiento de Goalbal (TLAN).

Nota: TLAN - Tiempo de Lanzamiento; ENV - Envergadura; CMS - Comprometimient Miembro superior; $1 \mathrm{RMe}$ - Repetición Máxima Estimada; AMB - Lanzamiento del Baló Medicinal; \%GC - Grasa Corporal.

\section{Discusión}

El objetivo del presente estudio fue investigar el perfil de los atletas de Goalball chilenos como también identificar la asociación y relación entre las variables antropométricas, de composición corporal y fuerza con el lanzamiento del balón de Goalball (TLAN).

En relación a los datos antropométricos, el estudio observó valores promedios de 23,2 $\pm 4,1 \%$ de GC. Estos datos representan valores inferiores a los obtenidos por atletas de

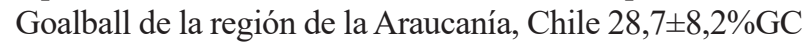
(Valdés, Godoy \& Herrera, 2014) y a los atletas de la selec-

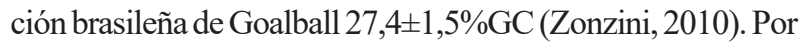
otro lado, nuestros valores fueron superiores en relación a los obtenidos por Rodrigues y Bastos (2007) de atletas brasileños de diferentes niveles $(20,4 \pm 4,4 \% \mathrm{GC})$ y cuando com- paramos el estudio más reciente, realizado por Scherer, Karasiak, Silva, \& Petroski (2012), el cual evaluaron a atletas de clubes amateur brasileños $(16,6 \pm 5,2 \% \mathrm{GC})$, nuestro estudio también presenta valores superiores.

Molik, Morgulec, Kosmol, Perkowski \& Bednarczuk (2015) realizaron un estudio con atletas de sexo masculino de Goalball de élite. El estudio fue desarrollado con el objetivo de determinar la relación entre las características antropométricas de envergadura y rendimiento deportivo según el grado de visión. El valor promedio fue de un $184,1 \pm 7,3 \mathrm{~cm}$, superiores a los alcanzados por nuestro estudio (174,3 $\pm 3,9 \mathrm{~cm})$. Según los autores (Molik et al., 2015), las variables antropométricas, principalmente la envergadura, no mostraron una relación significativa con el rendimiento de juego, de esta manera en acciones ofensivas, no se esperaría una mayor trascendencia de esta variable y su relación con el rendimiento deportivo.

Al comparar estas evidencias, en nuestro estudio, la ENV presentó correlación inversamente proporcional y significativa con $1 \mathrm{RMe}(\mathrm{r}=-.74, \mathrm{p}=.021)$, pero analizada su determinación con el TLAN, los resultados fueron de $\mathrm{R}^{2}=.43$. De esa forma, corroboramos con Molik et al. (2015) que, se necesita una investigación adicional para lograr información más evidente para el éxito en la modalidad.

En relación a variables del rendimiento motor, (Marqués, Van den Tillaar, Vescovi, \& González-Badillo, 2007) relacionaron el lanzamiento del balón y la fuerza máxima (1-RMBP) en atletas de élite de hándbol, hallaron una correlación alta e inversamente proporcional, de acuerdo a la clasificación del coeficiente de correlación propuesta por Hopkins (2000), entre el tiempo de lanzamiento y 1-RMBP ( $\mathrm{r}=-.64)$. Corroborando con las afirmaciones antes mencionadas en nuestro estudio, el valor de correlación entre 1RMe y TLAN fue significativo e inversamente proporcional $(\mathrm{r}=-.80, \mathrm{p}=.008)$ en la determinación de causa y efecto entre las variables fue de $\mathrm{R}^{2}=.64$. Representando que los atletas que presentaron mayor fuerza máxima estimada (1RMe) lograron un lanzamiento en menor tiempo (TLAN).

Da Rocha(2007), establece que es fundamental realizar entrenamientos de fuerza con ejercicios específicos de la modalidad, de esta forma mejora la fuerza potencia y fuerza resistencia lo que haría el juego mucho más veloz y mejoraría la eficacia del lanzamiento. En tal sentido Morato(2012), afirma que la velocidad de lanzamiento del balón de Goalball tiene una influencia sustancial a la hora de anotar un gol. De este modo el entrenamiento de la potencia y la fuerza máxima parece ser el más consistente para aumentar el rendimiento del lanzamiento, ya que está relacionada a mayor producción de fuerza en menor tiempo posible.

En ese sentido, nuestro estudio observó una correlación muy alta y significativa $(r=.89, \mathrm{p}=.000)$ entre $1 \mathrm{RMe} y$ $\mathrm{AMB}$, lo que representa que la fuerza máxima influye en una mayor distancia de lanzamiento del balón medicinal. En un estudio realizado por Ikeda, Kijima, Kawabata, Fuchimoto \& Ito(2007), en el cual midieron la relación entre el rendimiento del lanzamiento de balones medicinales laterales y la capacidades físicas de fuerza máxima en las pruebas de Squat (1RMPS) y Bench Press (1RMBP) en atletas de diferentes disciplinas, en donde la correlación entre el 1RMBP y el lanzamiento lateral de balón medicinal arroja $(\mathrm{r}=.72)$, los autores 
sostienen que, a mayor fuerza máxima el desempeño en pruebas de lanzamiento con balón medicinal tendrán una mejor performance para los ejecutantes.

En relación a la longitud de la extremidad superior (CMS) con la fuerza máxima (1RMe) un estudio realizado por Caruso, Taylor, Lutz, Olson, Mason, Borgsmiller, \& Riner (2012) investigaron a un grupo de jóvenes, los cuales debían realizar press de banca con tres pesos diferentes. Concluyeron que la longitud del brazo es una de las variables que se relaciona con la fuerza. De igual forma, Hart, Ward \& Mayhew(1991), investigaron las correlaciones antropométricas del rendimiento de press de banca después del entrenamiento de resistencia, en donde concluyeron que, los sujetos con brazos más cortos obtuvieron mejores resultados en la elevación de press de banca. Esto se asemeja a nuestro estudio, donde se encontró una correlación alta y significativa, inversamente proporcional entre la longitud del brazo y el $1 \mathrm{RMe}(\mathrm{r}=-.72, \mathrm{p}=$ .027). Tal factor puede estar relacionado a una mayor ventaja mecánica generada por los atletas con las extremidades más cortas, lo cual serán capaces de ejercer grandes fuerzas (Kane $\&$ Sternheim, 2007), pero el CMS no presentó un factor determinante considerable para el TLAN $\left(\mathrm{R}^{2}=.43\right)$.

\section{Conclusiones}

Dentro de los principales hallazgos del presente estudio, se verificó una correlación significativa entre TLAN con 1RMe y una determinación de $64 \%$, lo que indica que los atletas que presentaron mayor fuerza máxima estimada, lograron un menor tiempo en el lanzamiento del balón de Goalball, de este modo el trabajo de esta cualidad beneficiaria la performance deportiva de los atletas. Por otro lado, las variables de CMS y AMB presentaron alta correlación con el test $1 \mathrm{RMe}$, pero no fueron determinantes para el rendimiento deportivo cuando fue asociado con el TLAN lo que condiciona que, la variable antropométrica en el lanzamiento no es determinante y la acción motriz de AMB no es capaz de representar el movimiento del lanzamiento. Como limitación del estudio podríamos apuntar una necesidad de una muestra mayor y de distintos niveles competitivos, con la finalidad de corroborar con los resultados obtenidos en el presente estudio.

\section{Agradecimientos}

Los autores declaran agradecimientos a los profesores y atletas del Club Renacer de San Pedro de la Paz y Club Renacer Concepción, por su colaboración y asistencia en la toma de datos. Su gran disposición hizo que este estudio se haya hecho realidad.

\section{Referencias}

Amarante do Nascimento, M., Serpeloni-Cyrino, E., Yuzo-Nakamura, F., Romanzini, M., Cardoso-Pianca, H., \& Queiróga, M. (2007). Validação da equação de Brzycki para a estimativa de 1-RM no exercício supino em banco horizontal. Rev Bras Med Esporte, 13(1) - Jan/Fev, 2007. doi 10.1590/S1517-86922007000100011

Amorim, M., Corredeira, R., Sampaio, E., Bastos, T., \& Botelho, M. (2009). Goalball: uma modalidade desportiva de competição. Rev Port Cien Desp. 10(1) 221-229. doi: 10.5628/rpcd.10.01.221

Barrera, G. F. F., Torres, S. R. J., Díaz, C. C. J. C., Vásquez, D. C. D., Millar, D. F. V. (2020). Efectos de un programa de ejercicios de control postural en el equilibrio corporal y precisión de lanzamiento en tiro con arco en categoría infantil y cadetes. Retos. Nuevas tendencias en Educación Física, Deporte y Recreación, n.37, 291-296. doi: 70956/45311

Bowerman, S., Davis, R., Ford, S., \& Nichols, D. (2011). Phases of movement of Goalball throw related to ball velocity. Insight: Research and Practice in Visual Impairment and Blindness 4(4), 153-159.

Caruso, J., Taylor, S., Lutz, B., Olson, N., Mason, M., Borgsmiller, J., \& Riner, R. (2012). Anthropometry as a Predictor of Bench Press Performance Done At Different Loads. Journal of Strength and Conditioning Research, 26(9), 2460-2467. doi: 10.1519/JSC.0b013e31823c44bb

Da Rocha, M. (2007). Treinamento de força aplicado ao goalball. (Trabalho de Conclusão de Curso). Universidade Estadual de Campinas, São Paulo, Brasil.

Durnin, J.V. \& Womersley, J. (1974). Body fat assessed from total body density and its estimation from skinfold thickness: measurements on 481 men and women aged 16 to 72 years. Br. J. Nutr, 32(1):77-97. doi: 10.1079/BJN19740060

Frutos, J. B. (2014). Biomechanic description of Hammer Throw. Retos. Nuevas tendencias en Educación Física, Deporte y Recreación, n.25, 124130. https://recyt.fecyt.es/index.php/retos/article/view/34496/18629

Gómez, D. \& Tosim, A. (2016). Manual para entrenadores de goalball. USAID: Bogotá.

Guedes, D. P., \& Guedes, J. E. R. P. (2006). Manual praitico para avaliac'aPo em educac'apo fiìsica. Saధo Paulo, Brasil: Manole.

Guzmán, K. S., Fonseca, A. S., Jiménez, J. M. (2016). Acute effect of exergame practice on performance in basketball throw. Retos. Nuevas tendencias en Educación Física, Deporte y Recreación, n.29, 58-60. https://recyt.fecyt.es/ index.php/retos/article/view/36736/21767

Hart, C., Ward, T., \& Mayhew, J. (1991). Anthropometric correlates of bench press performance following resistance. Training, Sports Medicine, Training and Rehabilitation, 2(2), 89-95. doi: 10.1080/15438629109511904

Hernández, P. J. B., Lara, E. R., Iturriaga, F. M. A. (2017). Relationship among anthropometric parameters, maximal grip and throwing velocity in youth water polo players. Retos. Nuevas tendencias en Educación Física, Deporte y Recreación, 31(1), 212-218.

Hopkins, W. G. (2000). Correlation coefficient: a new view of statistics. Recuperado de: http://www.sportsci.org/resource/stats/correl.html

Ikeda, Y., Kijima, K., Kawabata, K., Fuchimoto, T. \& Ito, A. (2007) Relationship between side medicine-ball throw performance and physical ability for male and female athletes. European Journal of Applied Physiology, 99(1):47-55. doi: 10.1007/s00421-006-0316-4

ISAK. (2011). Protocolo internacional para la valoración antropométrica 2011. Recuperado de: https://es.scribd.com/document/375628061/ProtocoloAntropometria-2011-ISAK

Jimenez-Olmedo, J. M., Espina-Agullo, J . J., Manchado, C. (2017). Historical Analysis of throwing effectiveness in male handball. Retos. Nuevas tendencias en Educación Física, Deporte y Recreación, n.32, 228-232. https://recyt.fecyt.es/index.php/retos/article/view/56059/33852

Kane, J., \& Sternheim, M. (2007). Física. ( $2^{\mathrm{a}}$ ed.). Madrid, España: Reverté. Marqués, M., Van den Tillaar, R., Vescovi, J., \& González-Badill, J. (2007). Relationship Between Throwing Velocity, Muscle Power, and Bar Velocity Durin Bench Press in Elite Handball Players. International Journal of Sports Physiology and Performance, 2(4), 414-422. doi: 10.1123/ ijspp.2.4.414

Martínez, E. J. (2002). Pruebas de aptitud física. Barcelona, España: Editorial Paidotribo.

Molik, B., Morgulec, N., Kosmol, A., Perkowski, K., Bednarczuk, G., Skowronski, W., Gomez, M., Koc, K., Rutkowska, I. \& Szyman, R. (2015). Game Performance Evaluation in Male Goalball Players. Journal of Human Kinetics volumen, 48, 43-51. doi: 10.1515/hukin-2015-0090

Morato, M. (2012). Análise do jogo de Goalball: Modelação e interpretação dos padrões de jogo nas Paralimpiadas de Pequim 2008. (Tese de Doutorado). Universidad Estadual de Campinas, Brasil.

Pereira, M., Simões, M., \& Almeida, J. J. G. (2012). Os processos autoorganizacionais do Goalball. Rev. Bras. Ciênc. Esporte, Florianópolis; 34(3), p. 741-760. doi: 10.1590/S0101-32892012000300015.

Rodrigues, J., \& Bastos, T. (2007). Caracterização dos hábitos de ingestão nutricional e composição corporal de atletas masculinos praticantes de goalball. Arquivos em Movimento 3(2), 3-17.

Sanz, D., \& Reina, R. (2014). Actividades físicas y deportes adaptados para personas con discapacidad. Barcelona, España: Editorial Paidotribo.

Scherer, R., Karasiak, F., Silva, S., \& Petroski, E. (2012). Morphological profile of Goalball athletes. European Journal of Human Movement, 28(1), 1-132.

Siri, W. E. (1956). Gross Composition of the Body. Advances in Biological and Medical Physics, 4, p.239-80. doi: 10.1016/B978-1-4832-31105.50011-X

Valdés, P., Godoy, A., \& Herrera, T. (2014). Somatotype, Body Composition, Nutritional State and Physical Condition in People with Visual Impairment Who Practice Goalball. International Journal of Morphology, 32(1), 183189. doi: $10.4067 / \mathrm{S} 0717-95022014000100031$

Zonzini, D. (2010). Perfil somatotípico e composição corporal em atletas da seleção Brasilera masculina de Goalball. 2010. 29f. (Trabalho de Conclusão de Curso). Universidade Estadual de Campinas, Campinas, Brasil. 